\title{
Video Article \\ Preparation and Culture of Rat Lens Epithelial Explants for Studying Terminal Differentiation
}

\author{
Peggy S. Zelenka ${ }^{1}$, Chun Y. Gao ${ }^{1}$, Senthil S. Saravanamuthu ${ }^{1}$ \\ ${ }^{1}$ Laboratory of Molecular and Developmental Biology, National Eye Institute (NEI), National Institutes of Health (NIH) \\ Correspondence to: Peggy S. Zelenka at zelenkap@nei.nih.gov \\ URL: https://www.jove.com/video/1519 \\ DOI: doi:10.3791/1519
}

Keywords: Cellular Biology, Issue 31, lens, differentiation, FGF, rat

Date Published: 9/22/2009

Citation: Zelenka, P.S., Gao, C.Y., Saravanamuthu, S.S. Preparation and Culture of Rat Lens Epithelial Explants for Studying Terminal Differentiation. J. Vis. Exp. (31), e1519, doi:10.3791/1519 (2009).

\section{Abstract}

The anterior surface of the ocular lens is covered by a monolayer of epithelial cells, which proliferate in an annular zone underlying the ciliary body. Following division, these cells migrate posteriorly, where FGF diffusing from the retina induces them to differentiate into a posterior array of elongated lens fiber cells, which compose the bulk of the lens. Differentiation of lens epithelial cells into lens fibers can be induced in vitro by culturing explants of the central region of the anterior epithelium in the presence of FGF-2. Explants are prepared from lenses of neonatal rats by removing the lens from the eye and grasping the lens capsule on the posterior side with dissecting tweezers. The posterior capsule is then gently torn open and pressed down into the plastic bottom of a tissue culture dish. The peripheral regions of the explant are removed with a scalpel and the central area is then cultured in the presence of $100 \mathrm{ng} / \mathrm{ml} \mathrm{FGF-2} \mathrm{for} \mathrm{as} \mathrm{long} \mathrm{as} \mathrm{2-3} \mathrm{weeks,} \mathrm{depending} \mathrm{on} \mathrm{the} \mathrm{parameters} \mathrm{to} \mathrm{be} \mathrm{studied.} \mathrm{Since}$ epithelial cells in cultured explants differentiate in approximate synchrony over a period of days to weeks, the time course of signaling and gene expression can be determined using molecular, biochemical, and pharmacological techniques. Immunofluorescence microscopy is a powerful adjunct to these methods as it demonstrates the subcellular localization of proteins of interest and can reveal the physiological consequences of experimental manipulations of signaling pathways.

\section{Video Link}

The video component of this article can be found at https://www.jove.com/video/1519/

\section{Protocol}

\section{Part 1: Removal of lenses}

\section{Materials and reagents:}

1. Micro-dissecting scissors, curved, blunt tips, (such as.RS-5983, Roboz Surgical Instrument Co., Inc, Gaithersburg, MD); micro-dissecting tweezers, curved tip (such as Roboz \#RS5137).

2. Suspension medium: Medium 199 containing $0.1 \%$ bovine serum albumin, 100 units $/ \mathrm{ml}$ penicillin, $100 \mu \mathrm{g} / \mathrm{ml}$ streptomycin, $2.5 \mu \mathrm{g} / \mathrm{ml}$ Amphotericin B. Reagents are available from Invitrogen, Carlsbad, CA.

\section{Procedure:}

1. Euthanize newborn rats (aged 2-4 days) in accordance with guidelines provided by the National Institutes of Health, Bethesda, MD.

2. Remove the eyelids with surgical scissors. Press gently with curved tweezers on opposite sides of the eye socket to force the eye to bulge outward. Make a small incision on the posterior side of the eye with the scissors. By pressing with tweezers against the side of the eye opposite the incision, the lens and a small amount of attached vitreous body can then be forced out through the rupture, allowing the lens to be picked up with curved tweezers. Care should be taken not to break the lens capsule.

3. Use the curved tweezers to transfer the lenses to a $60 \mathrm{~mm}$ plastic tissue culture dish containing $5 \mathrm{ml}$ warm, sterile suspension medium.

\section{Part 2: Microdissection of explants}

\section{Materials and reagents:}

1. Micro Dissecting Tweezers, $0.1 \mathrm{~mm}$ tips, (such as Roboz RS-4976). We use Dumostar alloy because of the flexibility of the tip, but other steel alloys are also acceptable. Tweezers with tips thinner than $0.1 \mathrm{~mm}$ are not recommended, as they tend to break or bend during this procedure.

2. Suspension medium (see Part 1) 
3. Unsupplemented Ham's F12 medium or Medium 199 (Invitrogen, Carlsburg, CA)

\section{Procedure:}

1. The following steps should be carried out in a clean, draft-free environment using sterile dissecting tools and sterile medium.

2. Using a stereo-dissecting microscope, clean the lenses of any adhering tissue with the $0.1 \mathrm{~mm}$ tip tweezers and transfer them to a second $60 \mathrm{~mm}$ culture dish containing $5 \mathrm{ml}$ sterile suspension medium (this step helps to reduce contamination). With the tweezers, transfer the desired number of lenses to a $35 \mathrm{~mm}$ culture dish containing $5 \mathrm{ml}$ sterile, unsupplemented medium. (We often use Ham's F12 (Invitrogen) for this step, because the lower dye concentration makes dissection easier; however, Medium 199 is also acceptable. No antibiotics or other additions are needed during this step.) As many as 12 explants can be made in the center of a dish this size. However, a $35 \mathrm{~mm}$ dish should be used even if fewer explants are to be made, because the dissecting tools cannot be manipulated easily in a smaller dish. Transfer the dish containing the remaining lenses to a tissue culture incubator at $37^{\circ} \mathrm{C}$ until they are needed.

3. Identify the posterior side of the lens. There are many ways to recognize this: 1) The posterior is rounder than the anterior, which is slightly flattened; 2) Newborn rat lenses form cold cataracts as they cool to room temperature during the dissection. If the cold cataract has not completely filled the lens, the posterior side is the side farthest from the opaque region. If the opacity has filled the lens, warming the dish to $37^{\circ} \mathrm{C}$ for a few minutes will reverse it. The posterior side can be identified as the cataract reforms upon cooling. 3) Vestiges of the tunica vasculosa lentis may be visible on the posterior side. 4) The posterior suture may be visible on the posterior side. Identifying the posterior side correctly is essential, since this is where the capsule will be opened. Opening the lens on the anterior side will tear the epithelium.

4. Once the posterior side has been identified, turn it upward and grasp the lens in the left tweezers (for a right-handed worker). Then pinch the posterior capsule with the right tweezers to produce a small fold.

5. While holding the posterior capsule with the right tweezers, grasp the fold of capsule with the left tweezers and pull the two pairs of tweezers in opposite directions to make a small tear in the capsule.

6. While grasping the edge of the retracting capsule with the tweezers, pull it downward, first on one side, then on the other, pressing it into the plastic of the dish with the tweezers. Repeat this several times, moving all around the equator of the lens, until the capsule is firmly attached to the plate at many points. Holding the capsule in place with the left tweezers, gently rock the fiber mass with the right tweezers to break the attachment between the fiber cells and epithelial cells at the lens equator. Then push the fiber mass away, rolling it off the capsule/epithelium, which remains attached to the bottom of the dish. Continue this process with all the lenses in the dish. Remove and discard the fiber masses (or save them frozen as a source of lens proteins for other studies).

\section{Part 3: Microdissection and culture of central explants}

\section{Materials and reagents:}

1. Sterile disposable scalpel, \#15 blade, (Cincinnati Surgical Co. Cincinnati, $\mathrm{OH}$ )

2. Sterile phosphate buffered saline with calcium and magnesium (Invitrogen, Carlsbad, CA)

3. Culture medium: Suspension medium containing 100ng/ml FGF-2 (Sigma-Aldrich, Inc. St Louis, MO).

\section{Procedure:}

1. Using a sterile scalpel, trim away the peripheral epithelium (PE), which contains cells in the early stages of differentiation (Figure $1 \mathrm{~A})$, leaving a central square approximately $2.0 \mathrm{~mm}$ on a side, consisting of central epithelial cells only (CE) (Figure 1B).

2. Transfer the dish containing the central explants to a biosafety cabinet. Wash 3 times with sterile PBS containing calcium and magnesium and once with $1 \mathrm{ml}$ of fresh, equilibrated $\left(37^{\circ} \mathrm{C}, 5 \% \mathrm{CO}_{2}\right)$ suspension medium. These washes greatly reduce the likelihood of contamination.

3. Add $2 \mathrm{~mL}$ of culture medium to induce differentiation ${ }^{1}$, and place the explants in a humidified, tissue culture incubator at $37^{\circ} \mathrm{C}, 5 \% \mathrm{CO}_{2}$. Culture periods may be as short as a few hours or extend as long as two to three weeks, depending on the parameter being studied. Medium should be changed every two to three days.

\section{Part 4: Harvesting of explants for analysis of events associated with differentiation}

\section{Harvesting explants for analysis of protein or RNA}

\section{Materials:}

1. Microdissecting tweezers

2. SDS Lysis Buffer or RNA-later

\section{Procedure:}

1. At the end of the incubation period, remove the culture medium.

2. Using the stereo-dissecting microscope, gently loosen the edges of each explant.

3. Lift each explant with the tweezers and transfer it to a tube containing about 100 $\mu$ I SDS lysis buffer (for protein analysis) or RNA-later (for RNA analysis). Agitate the tip of the tweezers in the solution to ensure that the explant does not stick to the tweezers.

\section{Harvesting explants for immunofluorescence}

\section{Materials and reagents:}

1. Phosphate buffered saline with calcium and magnesium (Invitrogen, Carlsbad, CA)

2. Phosphate buffered saline (without calcium and magnesium) (Invitrogen, Carlsbad, CA)

3. $4 \%$ paraformaldehyde (Boston Bioproducts, Worcester, MA) 
4. Microdissecting tweezers

5. Hydrophobic marking pen

6. Glass microscope slides

7. $0.25 \%$ Triton $\mathrm{X}-100$ in phosphate buffered saline.

\section{Procedure:}

1. Rinse explants briefly in PBS containing calcium and magnesium.

2. Fix tissues by adding $4 \%$ paraformaldehyde for 30 minutes at room temperature.

3. Remove the fixative and replace with phosphate buffered saline. Fixed explants become somewhat stiff, allowing them to be lifted and transferred to glass slides for immunostaining.

4. Place a small drop of PBS (without calcium and magnesium) on the slide to help position the tissue and prevent curling. Using microdissecting tweezers, lift the explant and insert it into the drop on the slide. Without touching the explant, carefully remove the liquid with a paper wick to flatten the explant on the glass and let the tissue dry in air at room temperature for 3 to 5 minutes.

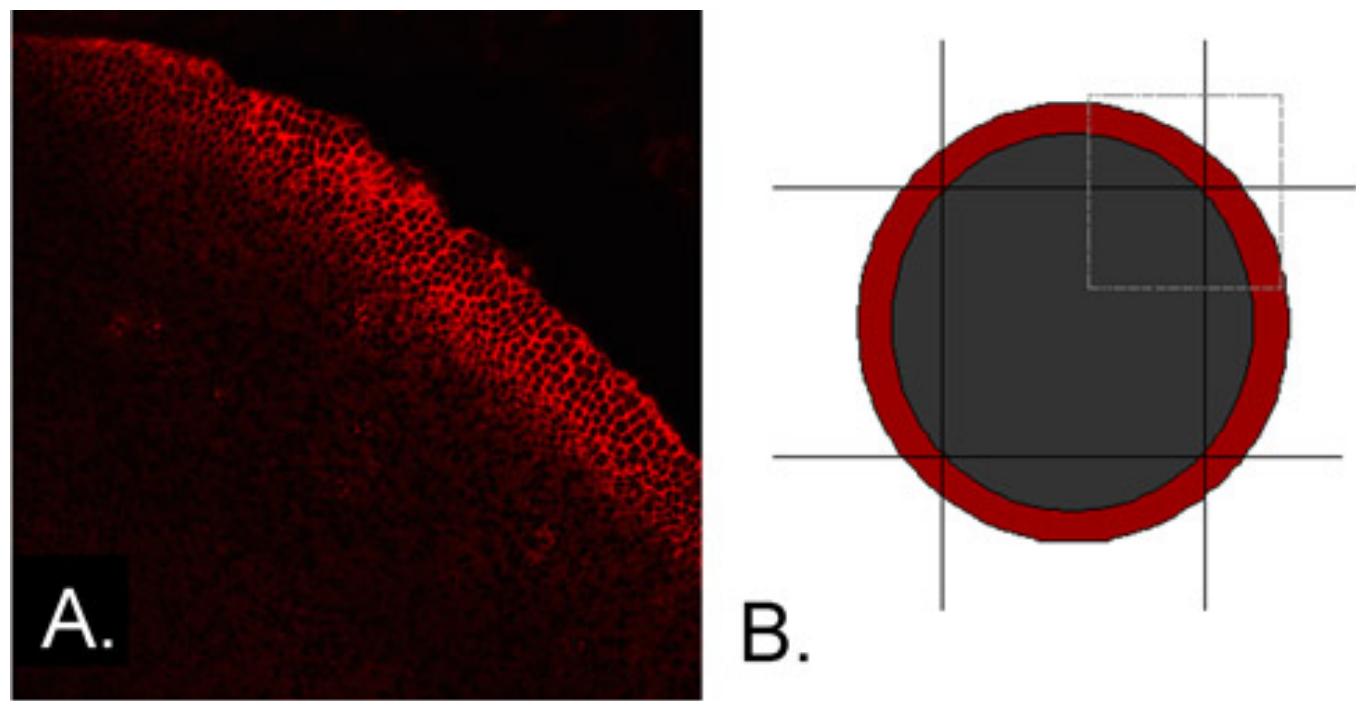

Figure $1 \mathrm{~A}$. The peripheral epithelium expresses differentiation-specific proteins. The explant shown was immunostained for $\mathrm{N}$-cadherin immediately after microdissection. Expression is seen in a band of cells in the peripheral epithelium, indicating that cells in this region have begun to differentiate. B. The peripheral epithelium can be trimmed away to remove cells that express $\mathrm{N}$-cadherin and other differentiationspecific proteins. The red annulus represents the location of cells that express $\mathrm{N}$-cadherin, while the small square outlined in gray represents the quadrant of the epithelium shown in panel $1 \mathrm{~A}$. The peripheral epithelium can be removed by four scalpel cuts, leaving a central square that contains only cells that have not yet begun to differentiate.

\section{Discussion}

The rat lens explant system has been successfully used by a number of laboratories to study terminal differentiation of lens epithelial cells to lens fibers ${ }^{21,3,4,5}$. When exposed to FGF-2 at $100 \mathrm{ng} / \mathrm{ml}$ the explants will begin to show changes in signaling within minutes ${ }^{6}$, with changes in morphology and gene expression appearing sequentially over several days ${ }^{3,4,5}$. The cultures remain viable for $2-3$ weeks if care is taken to prevent contamination.

The central explants described in this protocol are especially useful for studying the sequence of events associated with differentiation, since they contain few if any cells that express differentiation markers before FGF-2 is added ${ }^{1,5}$. The cells then differentiate synchronously, as a cohort, making it possible to follow the time course of signaling and transcriptional events associated with differentiation. Culturing explants for different lengths of time thus provides accurate temporal information about the sequence events. Specific inhibitors may be added to the culture medium to identify relevant signaling pathways. Explants may be used to analyze protein expression by SDS gel electrophoresis and immunoblotting or to analyze expression of specific mRNAs by RT-PCR. Protein yields range from 20-50 $\mu \mathrm{g} / \mathrm{explant}$ and RNA yield is approximately $200-600 \mathrm{ng} / \mathrm{explant}$, depending on the length of the culture period. We generally find that 5-6 explants per dish will provide sufficient protein or RNA for several assays. RNA from explants can also be used to prepare cDNA for assessing gene expression by microarray analysis, which can identify novel genes that may be critical for differentiation. Explants may also be transfected. Although transfection efficiency is generally low, it is sufficient for assaying reporter genes ${ }^{4 ; 7 ; 8}$. Immunofluorescence microscopy of the explants provides a useful adjunct to biochemical methods by determining the subcellular location of proteins of interest. Thus, the preparation and culture of rat lens explants provides a powerful system for studying terminal lens differentiation in mammals, which can complement in vivo techniques, such as generation of transgenic and knock-out mice.

Acknowledgements

The preparation of lens epithelial explants has been adapted from methods originated in the laboratory of Dr. John McAvoy ${ }^{9}$. This work is funded by the National Eye Institute, Intramural Research Program Z01-EY000238-22 


\section{References}

1. J.W. McAvoy and C.G. Chamberlain, Development (Cambridge, England) 107, 221 (1989).

2. M. T. Campbell and J. W. McAvoy, Exp Eye Res 39 (1), 83 (1984).

3. F. J. Lovicu and J. W. McAvoy, Exp Eye Res 49 (3), 479 (1989).

4. N. Golestaneh, J. Fan, R. N. Fariss et al., J Biol Chem 279 (30), 31813 (2004).

5. Senthil S. Saravanamuthu, Chun Y. Gao, and Peggy S. Zelenka, Developmental Biology In Press, Accepted Manuscript.

6. F. J. Lovicu and J. W. McAvoy, Development (Cambridge, England) 128 (24), 5075 (2001).

7. R. P. Dirks, H. J. Kraft, S. T. Van Genesen et al., Eur J Biochem 239 (1), 23 (1996).

8. Y. Yang, T. Stopka, N. Golestaneh et al., The EMBO journal 25 (10), 2107 (2006).

9. J. W. McAvoy and V. T. Fernon, Curr Eye Res 3 (6), 827 (1984). 